\title{
Classification of oral pigmented anaerobic bacilli by pyrolysis mass spectrometry and biochemical tests
}

\author{
J. T. MAGEE*, J. M. HINDMARCH, B. I. DUERDEN* and L. GOODWIN
}

Department of Experimental and Clinical Microbiology, University of Sheffield Medical School, Beech Hill Road, Sheffield S10 2RX

\begin{abstract}
Summary. Clinical (66) and reference (5) strains of pigmented gram-negative anaerobic bacilli, identified as Prevotella intermedia (47), Pr. melaninogenica (1), Pr. corpora (8), Porphyromonas asaccharolyticus (12), $P$. endodontalis (1) and $P$. gingivalis (2), were examined by pyrolysis mass spectrometry (PMS) and in conventional tests. Numerical classification based on conventional test reaction patterns (CTRPs) resolved five clusters, four comprising strains identified as $P r$. intermedia, Pr. corpora, Pr. melaninogenica, and $P$. gingivalis respectively, and one comprising strains identified as $P$. asaccharolyticus and $P$. endodontalis. Numerical classification based on PMS showed a similar division, with decreasing homogeneity of chemical composition in the order Pr. intermedia, Pr. corpora, P. asaccharolyticus, which agreed with the order of homogeneity in CTRPs. PMS clusters corresponding to the genus Porphyromonas were clearly distinct from those of the genus Prevotella. PMS and CTRP classification disagreed on cluster membership for six strains. PMS identification from blind challenge sets was in agreement with conventional identification for 64 of 67 strains.
\end{abstract}

\section{Introduction}

Gram-negative anaerobic bacilli that produced black or brown colonies on blood-containing media were first described by Oliver and Wherry ${ }^{1}$ and called Bacterium (later Bacteroides) melaninogenicus. This species encompassed all pigmented bacteroides but was subsequently divided into three subspecies. ${ }^{2}$ Subsequently the asaccharolytic strains were removed, first to a distinct species, B. asaccharolyticus, ${ }^{3}$ later as a subgroup of species within the Bacteroides ${ }^{4}$ and then to a separate genus, Porphyromonas, comprising three species, $P$. asaccharolyticus, $P$. endodontalis and $P$. gingivalis. ${ }^{5}$ The saccharolytic pigmented strains remained members of the melaninogenicus-oralis group of Bacteroides which included pigmented and nonpigmented strains; but these too were later separated from the genus Bacteroides sensu stricto $^{8}$ (formerly the fragilis group of Bacteroides) as a new genus, Prevotella. ${ }^{7}$ The pigmented Prevotella spp. comprise $P r$. intermedia, Pr. melaninogenica, Pr. corpora, Pr. loescheii and Pr. denticola. These changes were largely the result of DNA homology studies.

Pigmented bacteroides are members of the normal flora of the mouth, ${ }^{8-10}$ vagina ${ }^{11}$ and colon, ${ }^{12}$ and are also implicated in a range of purulent and necrotising

Received 21 May 1991; accepted 6 Sept. 1991.

* The present address to which correspondence should be sent: Department of Medical Microbiology and Public Health Laboratory, University of Wales College of Medicine, Heath Park, Cardiff CF4 4XN. infections at these sites. ${ }^{13-17}$ In the mouth they are found in the gingival crevice in both health and disease. ${ }^{9}$ Pr. melaninogenica is part of the normal commensal gingival flora ${ }^{9}$ and appears to be of little pathological significance, ${ }^{10}$ whereas $P r$. intermedia is more commonly associated with acute necrotising gingivitis and chronic periodontal disease. ${ }^{18-20} P$. gingivalis is linked with severe, rapidly progressive periodontitis and generalised juvenile periodontal disease. ${ }^{15,21} P$. asaccharolyticus has not been associated with oral colonisation or disease, but in a study of the gingival flora in patients with adult periodontal disease $^{22}$ we isolated large numbers of pigmented strains, about half of which appeared asaccharolytic in rapid tests, ${ }^{23-25}$ produced small amounts of n-butyric acid, and were identified as $P$. asaccharolyticus. Most of the saccharolytic strains were identified as $P r$. intermedia. Similar results were obtained in a subsequent longitudinal study of adult periodontal disease. ${ }^{26}$ The work described here was done partly because of doubts as to the validity of these identifications as $P$. asaccharolyticus, based solely on rapid tests.

Pyrolysis mass spectrometry (PMS) is a rapid characterisation method that has been used in classification, identification and typing of microorganisms. ${ }^{27,28}$ The spectra produced reflect cell chemical composition as 'fingerprint' or pattern data with a complex statistical structure. Analysis of these data requires multivariate statistical strategies. However, modern statistical program suites available for micro- and mini-computers can be used to reduce raw 
spectral data to statistically meaningful strain similarities, for classification, for typing, and to identify spectra of unknown strains by comparison with a concurrently produced database of spectra for named strains. The approach is particularly relevant in studies of genera where characterisation is difficult because strains are "biochemically unreactive" or require prolonged incubation to yield readable results. ${ }^{28}$ The identification problem described above seemed a fitting application, which would also extend our investigations into traditional and PMS numerical classification of the anaerobic gram-negative bacilli. ${ }^{29-31}$

\section{Materials and methods}

\section{Bacterial strains}

The 71 strains of pigmented gram-negative anaerobic bacilli tested comprised dental (53), vaginal (4) and wound (9) isolates, together with the type strains Pr. intermedia NCTC 9336, P. asaccharolyticus NCTC 9337, P. gingivalis NCTC 11834, P. endodontalis HG 370 and the collection strain $P$. gingivalis W50. These were preserved at $-70^{\circ} \mathrm{C}$ in glycerol $15 \%$ broth, and cultured on $\mathrm{BM}$ agar $^{32}$ at $37^{\circ} \mathrm{C}$ in an atmosphere of $\mathrm{H}_{2}$ $10 \%, \mathrm{~N}_{2} 80 \%, \mathrm{CO}_{2} 10 \%$ for $48 \mathrm{~h}$ before testing.

\section{Conventional tests}

The strains were examined in a range of conventional tests, only 10 of which gave reproducible results relevant in differentiation. These were: production of acid from glucose, lactose and sucrose ${ }^{24,25}$ production of indole, ${ }^{24,25} \alpha$-fucosidase, $\alpha$-glucosidase and $\beta$-Nacetyl glucosaminidase ${ }^{33}$ agglutination of sheep erythrocytes $;^{34}$ and production of n-butyric and phenyl acetic acids, ${ }^{35}$ detected by gas-liquid chromatography. ${ }^{36}$ Tests which proved non-discriminatory or irreproducible were: tolerance to sodium taurocholate, gentian violet and Victoria blue 4R; resistance to penicillin ( 2 units), neomycin $(1000 \mu \mathrm{g})$, kanamycin $(1000 \mu \mathrm{g})$ and rifampicin $(15 \mu \mathrm{g})$ disks $;{ }^{23-25}$ gas-liquid chromatography for non-volatile fatty acids $;^{36}$ and fluorescence under UV illumination. ${ }^{4,37}$

Conventional identification was based on comparison of conventional test reaction patterns (CTRPs) with those of type strains of the currently accepted species. The UPGMA hierarchical clustering strategy $^{38}$ and simple matching coefficient $\left(\mathrm{S}_{\mathrm{sm}}\right)^{39}$ were used to produce a numerical classification based on the 10 tests listed above, which gave the dendrogram shown in fig. 1.

\section{Pyrolysis mass spectrometry}

PMS methods and analysis strategies were as described previously. ${ }^{29-31,40}$ Briefly, the 71 strains, plus two duplicates (one each of Pr. intermedia and $P$. asaccharolyticus) were assigned blind numeric codes. Four replicate foils were prepared for each of these 73 "strains". The replicate foils of each strain were divided between four batches, each with a different randomised strain sequence. The four batches of foils were analysed over 2 days on a PYMS 200X automated pyrolysis mass spectrometer (Horizon Instruments, Heathfield, Sussex). Immediately before each batch run, six foils of three strains selected at random were pyrolysed and the spectra were discarded. In our experience, this procedure limits excess irreproducibility in spectra produced early in a batch run.

The blind coding of the 73 "strains" examined was not broken until PMS classification was completed. In this study, discriminant analysis based on intensities of masses 50-120 yielded 11 canonical discriminant function axes (CDFAs) describing $84.6 \%$ of interstrain discrimination. Spectral co-ordinates on these CDFAs were processed in UPGMA hierarchical clustering to produce a dendrogram (fig. 2). The squared Euclidian distance similarity coefficient $\left(D_{E}\right)$ used is an estimate of the $\chi^{2}$ probability ( 10 dof) that spectra of the strains compared were statistically indistinguishable. $^{28}$

PMS blind identification was performed as previously ${ }^{28-31,40}$ by inter-species discriminant analysis of normalised intensities for masses 50-120. Unduly optimistic estimates of identification error rates are likely to be obtained if spectra of the strain to be identified are included in the characterisation database. ${ }^{28}$ To avoid this, strains were sorted by conventional species identification, being put into the appropriate species set in the order of their initial random numerical blind coding. The strains within each species set were then numbered sequentially and two identification analyses were performed. In the first, all spectra of strains assigned odd species set numbers constituted the teaching set, describing the species characteristics in PMS, and all spectra of the even numbered strains were the blind challenge set. In the second analysis, membership of these sets was reversed. Spectra for the single strains representing $P r$. melaninogenica, $P$. endodontalis and $P$. gingivalis were omitted from these analyses, because no valid challenge sets could be assembled. Spectra of the six strains showing disagreement between PMS and conventional classification were included only in the challenge sets in both analyses. PMS species identifications for each spectrum in the challenge sets were noted, along with the consensus of the results for the four replicate spectra of each strain. If the identification results for a spectrum indicated a possible second choice at $\mathrm{p}>$ $5 \%$, the result was noted as equivocal and was ignored in the consensus strain identification.

\section{Results}

\section{Conventional classification}

At an $\mathrm{S}_{\mathrm{sm}}$ of $75-80 \%$, widely regarded as indicative of species discrimination, five clusters were resolved 


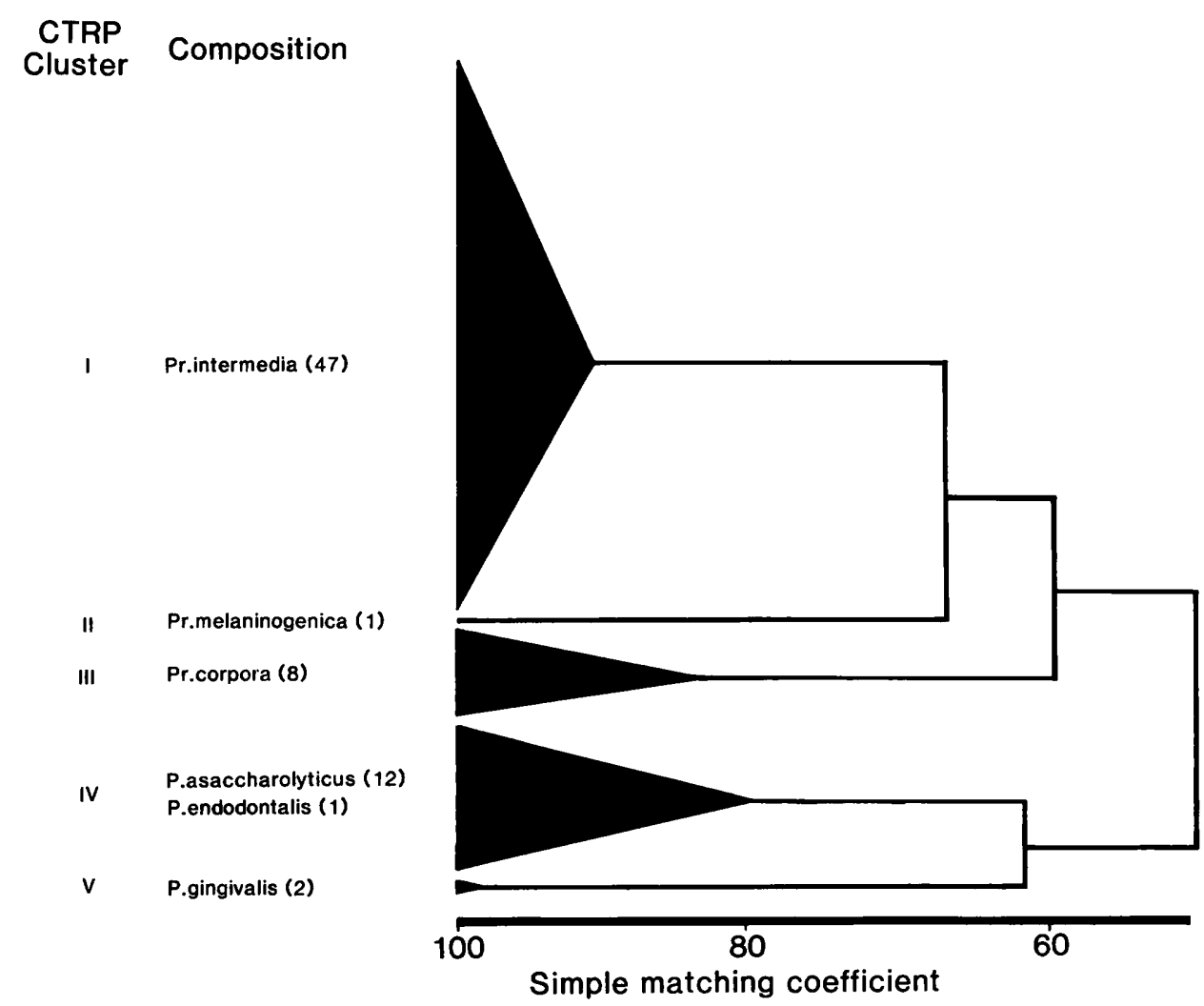

Fig. 1. A dendrogram representing a numerical classification of the strains based on conventional test reaction patterns. Tight correlation between conventional identification and cluster membership was expected, as the same tests contributed to both.

(fig. 1). Clusters I-III were distinct from clusters IV and $\mathrm{V}$ at $\mathrm{S}_{\mathrm{sm}}=0 \cdot 5$. All strains identified as Prevotella spp. were found in clusters I-III and all strains identified as Porphyromonas spp. were found in clusters IV and V. Cluster I comprised all 47 strains identified conventionally as Pr. intermedia, including the type strain NCTC 9336. Cluster II comprised a single strain, identified as Pr. melaninogenica. Cluster III comprised all eight strains identified as Pr. corpora.

Cluster IV comprised all 12 strains identified as $P$. asaccharolyticus, including the type strain NCTC 9337. The strain of $P$. endodontalis (ATCC 35406) was a single member outlier of this group. Cluster V comprised both strains identified as $P$. gingivalis (type strain NCTC 11834 and collection strain W50). Cluster I showed tight homogeneity of CTRPs, clusters III and IV were more diverse. Tight correlation between cluster membership and conventional identification was expected, because the same test results contributed to both.

\section{PMS classification}

Pairs for the two duplicated cultures were close neighbours $\left(\mathrm{D}_{\mathrm{E}}<80\right)$ in the dendrogram (fig. 2). Thirteen clusters were resolved at $D_{E}=600$. Cluster $A$ comprised 46 strains, 44 of which were identified conventionally as $P r$. intermedia, including the type strain NCTC 9336, and two as Pr. corpora. The single member cluster B comprised the strain identified as $P r$. melaninogenica. Cluster $\mathrm{C}$ comprised two strains, one identified as $\mathrm{Pr}$. intermedia and the other as $\mathrm{Pr}$. corpora. Clusters D and E comprised five strains identified as $P r$. corpora. Clusters $F$ and $G$ each comprised a single strain identified as Pr. intermedia. These clusters contained all strains identified as Prevotella spp. and were clearly distinct from the remaining clusters containing Porphyromonas spp.

Clusters H-J comprised the 12 strains identified as $P$. asaccharolyticus including the type strain NCTC 9337. Cluster $\mathrm{K}$ comprised the single strain of $P$. endodontalis (ATCC 35406), and clusters L and M comprised the collection strains of $P$. gingivalis W50 and NCTC 11834. Only six strains showed anomalous PMS clustering in comparison with conventional results; these are discussed below.

\section{Comparison of PMS and conventional classification results}

Of the six strains clustered by PMS in disagreement with conventional identification and classification results, three, identified conventionally as $\mathrm{Pr}$. corpora in cluster III, were found in PMS sub-groups A, A and $\mathrm{C}$ respectively. The remaining three, identified as $P r$. intermedia in cluster I, clustered in PMS groups $\mathrm{C}, \mathrm{F}$ and $\mathrm{G}$ respectively.

The level of CTRP diversity within each species and within the CTRP classification clusters was mirrored in the PMS classification. With the exceptions listed above, PMS group A showed tight homogeneity of chemical composition, corresponding to the tight 


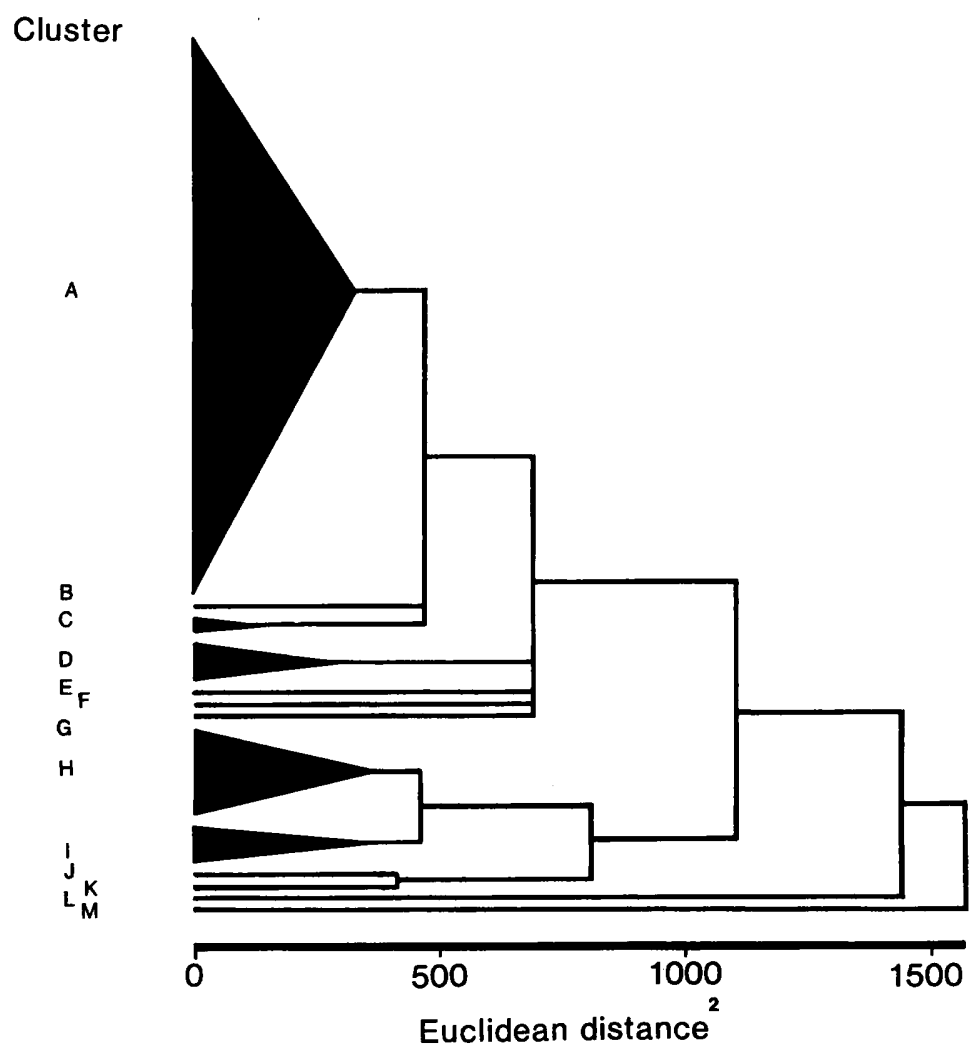

Fig. 2. A dendrogram representing a numerical classification of the strains based on pyrolysis mass spectrometry data. Cluster composition (conventional identification, number of strains) was: A, Pr. intermedia 44 plus Pr. corpora, 2; B, Pr. melaninogenica, $1 ;$ C, Pr. intermedia, 1 plus $\operatorname{Pr}$. corpora, 1; D and E, Pr. corpora, 4 and 1 respectively; F and $\mathbf{G}$, Pr. intermedia, 1 and 1 respectively; H-J, P. asaccharolyticus, 8, 3 and 1 respectively; $\mathbf{K}, P$. endodontalis, $1 ; \mathbf{L}$ and $\mathbf{M}, P$. gingivalis, 1 and 1 respectively.

homogeneity of CTRPs for the Pr. intermedia strains of the equivalent conventional cluster I. Conventional cluster III (Pr. corpora) corresponded to the more diverse PMS clusters D and E, and showed wider diversity of CTRPs. P. asaccharolyticus showed a still wider CTRP diversity, corresponding to the wide divergence of PMS clusters $\mathbf{H}-\mathbf{J}$.

\section{PMS identification}

Of the 67 strains identified in PMS, 95.5\% yielded consensus results in agreement with conventional identification. Only three $(4.5 \%)$ showed disagreement. All three showed anomalous clustering in the PMS classification; two strains identified conventionally as Pr. corpora but clustering in PMS group A were identified as $P r$. intermedia, and one strain identified conventionally as Pr. intermedia but clustering in PMS group C was identified as Pr. corpora. The two strains comprising PMS clusters $F$ and $G$ were identified as $P r$. intermedia, in agreement with conventional identification, despite their anomalous clustering in PMS. Of the 280 individual spectra examined, $262(93.6 \%)$ were identified unequivocally in agreement with conventional identification, $14(5 \%)$ were identified to a species in disagreement with conventional identification (these included the four replicate spectra for each of the three strains identified in disagreement), three $(1 \cdot 1 \%)$ gave equivocal identifications, with either the first or second choice species in agreement with conventional identification, and one $(0.3 \%)$ gave an equivocal identification with neither first nor second choice species in agreement.

\section{Discussion}

Clusters in the CTRP and PMS classifications were clearly identified with the currently recognised species and both classifications indicated major differences between the genera Prevotella and Porphyromonas. At species level, strains identified as Pr. corpora and as $P$. asaccharolyticus showed higher levels of compositional and biochemical heterogeneity than was found for the majority of strains identified as Pr. intermedia. However, the success in PMS identification indicated a cohesive core of compositional properties characteristic of each of these species.

By contrast, the two strains of $P$. gingivalis differed widely in PMS, but yielded identical results in conventional tests. Examination of a larger collection of Porphyromonas strains might resolve this problem, and throw light on the position of $P$. endodontalis, which appeared similar to $P$. asaccharolyticus in CTRPs and PMS.

There was over $90 \%$ agreement between PMS identification and that based on CTRPs, as in previous studies of Fusobacterium, ${ }^{29}$ coryneforms ${ }^{40}$ and the "Streptococcus milleri group". ${ }^{41}$ The only disagreement was in identification as Pr. corpora or Pr. 
intermedia. Conventional discrimination of these species can be difficult, and either CTRP or PMS identifications may have been erroneous for the few "mis-identified" strains. All strains identified as $P$. asaccharolyticus by rapid methods in our previous studies of oral flora ${ }^{22,26}$ were identified as $P$. asaccharolyticus by PMS, and none showed anomalous clustering in the PMS and CTRP classifications. The PMS identification results confirm that each of these three species shows a distinctive core of characteristic compositional features.

The possibility of exploiting PMS as a rapid identification system for gram-negative anaerobes seems attractive. The speed, throughput and disposables costs of PMS are suited to such an application. Wieten and his colleagues have outlined the successful use of PMS in routine identification for clinical isolates of mycobacteria. ${ }^{42,43}$ Because the long-term reproducibility of PMS remains poorly documented, they included a teaching set of named strains of mycobacteria with each batch of unidentified isolates, an approach which they term "operational fingerprinting' ${ }^{42}$

The biochemically unreactive nature of these organisms limited the number of tests used in the CTRP classification to 10. A far greater number of tests is recommended for numerical classification, and at least 30 tests showing discriminatory reactions are normally found for the more tractable aerobic genera of bacteria. With a larger number of tests, inter-strain similarity estimates are less affected by test errors, and tend to a stable figure, affected little by inclusion or exclusion of individual test results. The failure to find adequate numbers of discriminatory conventional tests has limited the application of numerical classification to the bacteroides and the progress in dividing this group into phenotypically and genotypically homogeneous species.

Clearly, reliable prediction of pathogenesis and other ecological properties is unlikely to be attained in a classification based on heterogenous species. The increasing complexity of the classification of the bacteroides may be irksome, but it is essential for rational work on pathogenesis. If the salmonellas and klebsiellas were viewed as one species and the shigellas and pseudomonads as another, there would be little hope of making sense of their pathogenicity. The bacteroides are probably as diverse as the enterobacteria, requiring a classification of similar complexity.

The introduction of techniques for genotypic classification of the bacteroides has allowed progress, but the low throughput of these techniques severely limits the number of strain comparisons that can be made. Often, only one or two strains are taken to represent a single species in such investigations. Phenotypic resemblance to these marker strains in a limited number of conventional tests may not be a reliable guide to genotypic homogeneity, raising the possibility that the currently postulated species structure may be incomplete. Although the results here do not suggest such anomalies, in a concurrent study of the non-pigmented bacteroides $^{31}$ several species appeared heterogeneous and two undescribed centres of variation were found.

The present throughput limitations of genetic methods suit them more to confirmation of phenotypic classifications than to exploratory work. What is required is a high throughput technique which can be applied to anaerobes and examines a large number of characters. Objective numerical studies for large strain collections would then give a rational framework for DNA hybridisation work. PMS is rapid, automated, with a high throughput and low running costs, and examines the large number of characters that contribute to total cell composition. Our work with other genera $^{30,31,40}$ has shown good correlations between PMS and CTRP classifications, and with DNA-DNA hybridisation data. ${ }^{41}$ PMS seems an approach well suited to the current needs of those interested in the taxonomy of anaerobes.

We thank Perkin Elmer, the trustees of the United Sheffield Hospitals Fund, Horizon Instruments and Trent Region Research Committee for their generous assistance in funding, loans of equipment and other help for our ongoing work on pyrolysis.

\section{References}

1. Oliver WW, Wherry WB. Notes on some bacterial parasites of the human mucous membranes. $J$ Infect Dis 1921; 28: 341-345.

2. Moore WEC, Holdeman LV. New names and combinations in the genera Bacteroides Castellani and Chalmers, Fusobacterium Knorr, Eubacterium Prévot, Propionibacterium Delwich, and Lactobacillus Orla-Jensen. Int J Syst Bacteriol 1973; 23: 69-74.

3. Finegold SM, Barnes EM. Report of the ICSB Taxonomic Subcommittee on Gram-negative Anaerobic Rods. Proposal that the saccharolytic and asaccharolytic strains at present classified in the species Bacteroides melaninogenicus (Oliver and Wherry) be reclassified in two species as Bacteroides melaninogenicus and Bacteroides asaccharolyticus. Int J Syst Bacteriol 1977; 27 : 388-391.

4. Coykendall AL, Kaczmarek FS, Slots J. Genetic heterogeneity in Bacteroides asaccharolyticus (Holdeman and Moore 1970) Finegold and Barnes 1977 (Approved Lists, 1980)

and proposal of Bacteroides gingivalis sp. nov. and Bacteroides macacae (Slots and Genco) comb. nov. Int J Syst Bacteriol 1980; 30: 559-564.

5. Shah HN, Collins MD. Proposal for reclassification of Bacteroides asaccharolyticus, Bacteroides gingivalis, and Bacteroides endodontalis in a new genus, Porphyromonas. Int J Syst Bacteriol 1988; 38: 128-131.

6. Shah HN, Collins MD. Proposal to restrict the genus Bacteroides (Castellani and Chalmers) to Bacteroides fragilis and closely related species. Int J Syst Bacteriol 1989; 39 : 85-87.

7. Shah HN, Collins MD. Prevotella, a new genus to include Bacteroides melaninogenicus and related species formerly classified in the genus Bacteroides. Int J Syst Bacteriol 1990; 40: 205-208.

8. Socransky SS, Gibbons RJ, Dale AC, Bortnick L, Rosenthal E, MacDonald JB. The microbiota of the gingival crevice area of man. I. Total microscopic and viable counts and counts of specific organisms. Arch Oral Biol 1963; 8: 275-280.

9. Slots J. Microflora in the healthy gingival sulcus in man. Scand $J$ Dent Res 1977; 85: 247-254. 
10. Duerden BI. The isolation and identification of Bacteroides spp. from the normal human gingival flora. $J$ Med Microbiol $1980 ; 13: 89-101$

11. Gorbach SL, Menda KB, Thadepalli H, Keith L. Anaerobic microflora of the cervix in healthy women. Am J Obstet Gynecol 1973; 117: 1053-1055.

12. Drasar BS, Shiner M, McLeod GM. Studies on the intestinal flora. Gastroenterology 1969; 56: 71-79.

13. Slots J. Subgingival microflora and periodontal disease. $J$ Clin Periodontol 1979; 6: 351-382.

14. Finegold SM. Anaerobic bacteria in human disease. New York, Academic Press. 1977.

15. van Steenbergen TJM, van Winkelhoff AJ, van der Velden $U$, de Graaff J. Taxonomy, virulence and epidemiology of blackpigmented Bacteroides species in relation to oral infections. Infection 1989; 17: 194-196.

16. Finegold SM. Intra-abdominal, genitourinary, skin and soft tissue infections due to non-sporing anaerobic bacteria. In: Phillips I, Sussman M (eds) Infection with non-sporing bacteria. Edinburgh, Churchill Livingstone. 1974: 160.

17. Thadepalli H, Gorbach SL, Keith L. Anaerobic infections of the female genital tract: bacteriologic and therapeutic aspects. Am J Obstet Gynecol 1973; 117: 1034-1040.

18. Slots J. Importance of black pigmented Bacteroides in human periodontal disease In: Genco RJ, Mergenhagan SE (eds) Host parasite interactions in periodontal diseases. Washington DC, American Society for Microbiology. 1982: 27.

19. van Winkelhoff AJ, van Steenbergen TJM, de Graaff J. The role of black pigmented Bacteroides in human oral infections. $J$ Clin Periodontol 1988; 15: 145-155.

20. Slots J, Listgarten MA. Bacteroides gingivalis, Bacteroides intermedius and Actinobacillus actinomycetemcomitans in human periodontal diseases. $J$ Clin Periodontal 1988; 15: 85-93.

21. Loesche WJ, Syed SA, Morrison EC, Laughton B, Grossman NS. Treatment of periodontal infections due to anaerobic bacteria with short-term treatment with metronidazole. $J$ Clin Periodontol $1981 ; 8: 29-44$.

22. Duerden BI, Goodwin L, O'Neil TCA. Identification of Bacteroides species from adult periodontal disease. $J$ Med Microbiol 1987; 24: 133-137.

23. Duerden BI, Holbrook WP, Collee JG, Watt B. The characterization of clinically important gram-negative anaerobic bacilli by conventional bacteriological tests. $J$ Appl Bacteriol 1976; 40: 163-188.

24. Duerden BI, Collee JG, Brown R, Deacon AG, Holbrook WP. A scheme for the identification of clinical isolates of gramnegative anaerobic bacilli by conventional bacteriological tests. J Med Microbiol 1980; 13: 231-245.

25. Rotimi VO, Faulkner J, Duerden BI. Rapid methods for identification of clinical isolates of gram-negative anaerobic bacilli. Med Lab Sci 1980; 37: 331-339.

26. Rawlinson A. The clinical and microbial changes during the treatment of periodontal disease with special reference to anaerobic gram-negative organisms. MDS thesis, University of Sheffield, 1990

27. Gutteridge CS, Vallis L, MacFie HJH. Numerical methods in the classification of micro-organisms by pyrolysis mass spectrometry. In: Goodfellow M, Jones D, Priest FG (eds) Computer-assisted bacterial systematics. (Special publications of the Society for General Microbiology 15.) London, Academic Press. 1985: 369-402.

28. Magee JT. Whole organism fingerprinting. In: Goodfellow $\mathbf{M}$, O'Donnell K (eds) The new bacterial taxonomy. London, Academic Press. 1991, in press.

29. Magee JT, Hindmarch JM, Bennett KW, Duerden BI, Aries RE. A pyrolysis mass spectrometry study of fusobacteria. $J$ Med Microbiol 1989; 28: 227-236.

30. Duerden BI, Eley A, Goodwin L, Magee JT, Hindmarch JM, Bennett $\mathrm{KW}$. A comparison of Bacteroides ureolyticus isolates from different clinical sources. $J$ Med Microbiol $1989 ; 29: 63-73$.

31. Magee JT, Yousefi-Mashouf R, Hindmarch JM, Duerden BI. A pyrolysis mass spectrometry study of the non-pigmented Prevotella species. J Med Microbiol 1992 (in press).

32. Holbrook WP, Ogston SA, Ross PW. A method for the isolation of Bacteroides melaninogenicus from the human mouth. $J$ Med Microbiol 1978; 11: 203-207.

33. Casals JB, Pringler N. Diagnostic tablets for bacterial identification. Rosco Diagnostica, Taastrup, Denmark. 1987

34. Slots J, Genco RJ. Direct hemagglutination technique for differentiating Bacteroides asaccharolyticus oral strains from nonoral strains. J Clin Microbiol 1979; 10: 371-373.

35. Kaczmarek FS, Coykendall AL. Production of phenylacetic acid by strains of Bacteroides asaccharolyticus and Bacteroides gingivalis sp. nov. J Clin Microbiol 1980; 12 . 288-290.

36. Deacon AG, Duerden BI, Holbrook WP. Gas-liquid chromatographic analysis of metabolic products in the identification of Bacteroidaceae of clinical interest. $J$ Med Microbiol $1978 ; 11$ : 81-99.

37. Brazier JS. A note on ultra-violet red fluorescence of anaerobic bacteria. J Appl Bacteriol 1986; 60: 121-126.

38. Rohlf FJ. Congruence of larval and adult classifications in Aedes (Diptera, Culicidae). Syst Zool 1963; 12: 97-117.

39. Sokal RR, Michener CD. A statistical method for evaluating systematic relationships. University of Kansas Science Bulletin 1958; 38: 1409-1438.

40. Hindmarch JM, Magee JT, Hadfield MA, Duerden BI. A pyrolysis-mass spectrometry study of Corynebacterium spp. J Med Microbiol 1990; 31 : 137-149.

41. Winstanley TG, Magee JT, Limb DI et al. A numerical taxonomic study of the "Streptococcus milleri group" based upon conventional phenotypic tests and pyrolysis mass spectrometry. J Med Microbiol 36: 149-155.

42. Wieten G, Haverkamp J, Meuzelaar HLC, Engel HWB, Berwald LG. Pyrolysis mass spectrometry: a new method to differentiate between the mycobacteria of the "Tuberculosis complex" and other mycobacteria. J Gen Microbiol 1981 ; 122 : 109-118.

43. Wieten G, Haverkamp J, Engel HWB, Berwald LG. Application of pyrolysis mass spectrometry to the classification and identification of mycobacteria. Rev Infect Dis 1981; 3 871-877. 